\title{
Different neuronal responses in the cochlear nucleus of a cat during classical and instrumental conditioning 1
}

\author{
EDWARD S. HALAS and JAMES $V$. \\ $B E A R D S L E Y$, University of North Dakota, \\ Grand Forks, N. Dak. 58201
}

Multiple-unit neuronal responses were recorded from the cochlear nucleus of a cat during classical and instrumental conditioning. Results indicate that different configurations of neuronal responses occurred during classical and instrumental conditioning.

Within the last several years, various investigators have attempted to correlate single- (O'Brien \& Fox, 1969a, b) and multiple-unit neuronal activity (Galin, 1965; Buchwald, Halas, \& Schramm, 1965, 1966) with classical conditioning. The positive results obtained in these studies would suggest that the recording of neuronal responses provides some useful information on the functions of various brain structures during conditioning. As an example, it was observed that the classical auditory pathway played a significant role during conditioning when an auditory stimulus was used as a CS, while the visual pathway was essentially nonfunctional (Buchwald, Halas, \& Schramm, 1966). The present study attempts to determine if there are any differences in the multiple-unit responses of the cochlear nucleus during classical and instrumental conditioning.

\section{METHOD}

The $S$ was an adult male cat with chronically implanted electrodes. The procedure for implanting an electrode in the dorsal cochlear nucleus has been described elsewhere (Buchwald, Halas, \& Schramm, 1966; Halas \& Beardsley, 1968). The multiple-unit neuronal activity was displayed on a Tektronix 565 oscilloscope and photographed with a Grass $\mathrm{C} 4$ camera. A modified version of the Weber \& Buchwald (1965) electronic integrator was used to count the frequency of the multiple-unit activity. The integration of the frequency count was displayed on the scope and photographed simultaneously with the neuronal activity. Pin lights mounted on the scope recorded the duration of the $\mathrm{CS}$ and US, which consisted of a $1,500 \mathrm{~Hz}$ tone paired with a mild electric shock to the hindpaw of the cat. The CS lasted $1.5 \mathrm{sec}$, and the US lasted $0.5 \mathrm{sec}$. The US overlapped the last third of the CS.

The $S$ was given 500 habituation trials to the tone at the rate of 39 trials per day ( 32 trials on the last day), followed by 800 trials of paired tone and shock during classical conditioning. The $S$ was then given 500 extinction trials followed by a 6-week rest period. After a second habituation of $\mathbf{5 0 0}$ trials, the $S$ was given 1,000 instrumental avoidance trials. If the $S$ lifted its hindpaw during the first $1.0 \mathrm{sec}$ of the tone, shock was not given. Five hundred extinction trials followed the avoidance training. Intertrial intervals ranged randomly from 20 to $50 \mathrm{sec}$.

\section{RESULTS}

The results clearly indicate that a different configuration of neuronal responses developed for instrumental as compared to classical conditioning. The neuronal responses are illustrated in Fig. 1. During habituation and the first 41 trials of classical conditioning, no behavioral or neuronal responses were observed (Traces $A$ and B). Starting with Trial 42, a positive sustained response was observed (Trace $\mathrm{C}$ ). Conditioned neuronal responses appeared $85 \%$ of the time from Trials 42 through 324 , while condtioned behavioral responses were observed on $34 \%$ of the trials. The configuration of the neuronal response remained essentially unchanged throughout the remainder of the classical-conditioning procedure (Traces D and E). From Trials 325 through 800 , neuronal responses occurred $78 \%$ of the time, while behavioral responses appeared $57 \%$ of the time. When the cat was extinguished, the neuronal response gradually diminished (Trace F). After extinction, the animal was given 6 weeks of rest followed by a second habituation period of 500 trials. Positive neuronal responses were observed in $21 \%$ of the habituation trials.

For the first 72 trials of instrumental conditioning, no neuronal or behavioral responses were observed (Trace $\mathrm{H}$ ). On Trial 73, a positive sustained response (Trace I) appeared. This response occurred $81 \%$ of the time from Trials 73 through 324 , while the conditioned behavioral response appeared $47 \%$ of the time. On Trial 325 , the response changed to a positive onset (Trace J) that was not sustained as in the earlier trials. From Trials 325 through 396, this onset response occurred $67 \%$ of the time. The behavioral response was elicited $73 \%$ of the time. Starting with Trial 397, the neuronal response evolved into a brief onset followed by a marked inhibition of the neuronal activity (Trace $\mathrm{K}$ ). This response persisted (65\%) through Trial 1,000 when the cat was extinguished. The behavioral response appeared $81 \%$ of the time. Extinction was also characterized by different responses. During early extinction, the previously observed complex conditioned neuronal response disappeared, and a positive sustained response (Trace $L$ ) appeared that was very similar to the response observed in Trace $I$. This response remained throughout extinction (500 trials).

DISCUSSION
Although the overt behavioral conditioned response (hindleg flexion) was essentially the same for both classical and instrumental conditioning, the neuronal responses were different for the two conditioning procedures. The neuronal response during classical conditioning was a positive sustained response that varied its amplitude some what but did not change its configuration. The neuronal response during instrumental conditioning revealed three different configurations during the training procedure. Rather interestingly, the first configuration to appear early in instrumental conditioning (Trace I) was very similar to the configuration that appeared in classical conditioning (Traces $C$, D, E). As instrumental training progressed, the configuration of the neuronal response changed. There is a considerable amount of behavioral data (Shapiro \& Miller, 1965) to suggest that the early stages of instrumental conditioning are identical to classical conditioning. The present neuronal data supports the position that the early phases of instrumental conditioning involve a classical-conditioning paradigm.

Furthermore, these results clearly indicate that the neural mechanism responsible for classical and instrumental conditioning involve the lower levels of the brain stem. What is perhaps more interesting is that the differentiation of classical and instrumental conditioning does occur so low in the brain stem. Whether this differentiation is mediated solely by neural mechanisms within the cochlear nucleus or is influenced by a feedback system from such higher structures as the cortex cannot be determined by the present data.

\section{REFERENCES}

BUCHWALD, J. S., HALAS, E. S., \& SCHRAMM S. Comparison of multiple-unit and EEG activity recorded from the same brain sites during behavioral conditioning. Nature, 1965 , 205, 1012-1014.

BUCHWALD, J. S., HALAS, E. S., \& SCHRAMM, $S$. Changes in cortical and subcortical unit activity during conditioning in chronic cats. Physiology \& Behavior, 1966, 1, 11-22.

GALIN, D. Background and evoked activity in the auditory pathway: Effects of noise-shock pairing. Science, 1965, 149, 761-763.

HALAS, E. S., \& BEARDSLEY, J. V. Specificity 


\section{CLASSICAL CONDITIONING}

TRACE A HABITUATION TRIAL 500
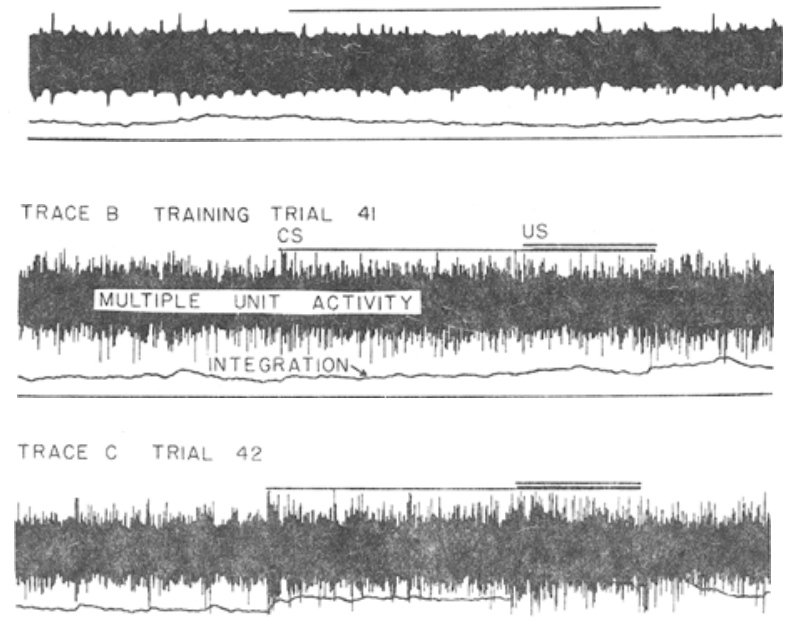

TRACE D TRIAL 325

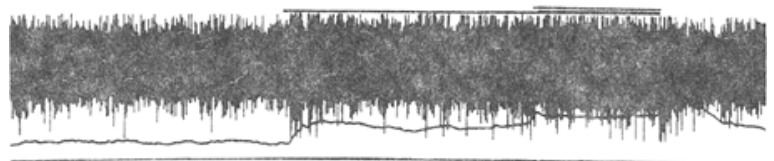

TRACE E TRIAL 600

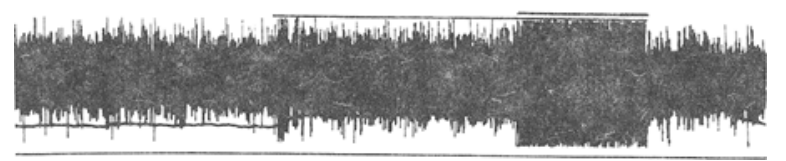

TRACE F EXTINCTION TRIAL 165
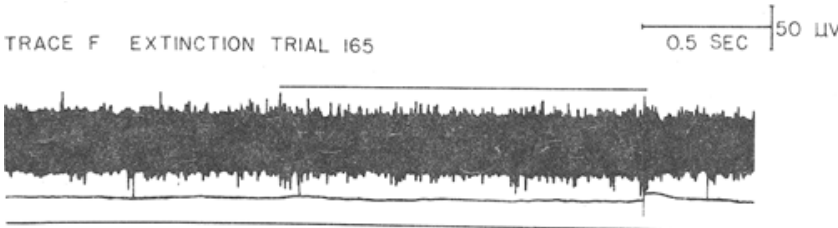

\section{INSTRUMENTAL CONDITIONING}

TRACE G HABITUATION TRIAL 500
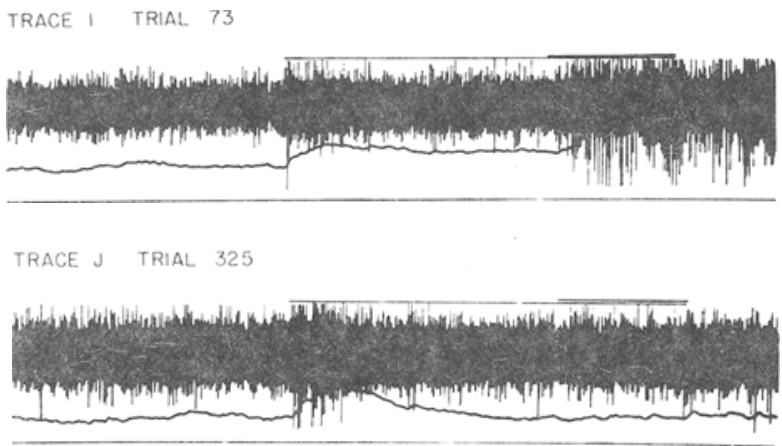

TRACE $K$ TRIAL 397

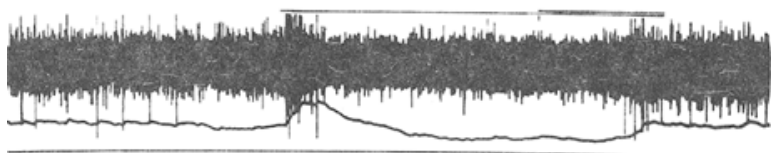

TRACE L EXTINCTION TRIAL 500

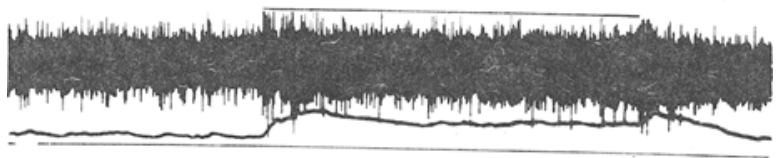

Fig. 1. Different configurations of neuronal responses recorded from the cochlear nucleus. The left half of the figure shows the neuronal responses recorded during habituation, classical conditioning, and extinction. The right half shows the neuronal responses recorded during habituation, instrumental conditioning, and ex tinction.

of multiple unit activity in the sensory nuclei of cats. Phy siology \& Behavior, 1968, 3, 275-279.

O'BRIEN, H. H., \& FOX, S. S. Single-cell activity in cat motor cortex: I. Modifications during classical conditioning procedures. Journal of Neurophysiology, 1969a, 32, 267-284.

O'BRIEN, J.H., \& FOX, S. S. Single-cell activity in cat motor cortex: II. Functional characteristics of the cell related to conditioning changes.
Journal of Neurophysiology, 1969b, 32, 285-296.

SHAPIRO, M. M., \& MILLER, T. M. On the relationship between conditioned and discriminative stimuli and between instrumental and consumatory responses. In W. F. Prokasy (Ed.), Classical conditioning: $A$ symposium. New York: Appleton-Century-Crofts, 1965 Pp. 269-301.
WEBER, D. S., \& BUCHWALD, J. S. A technique for recording and integrating multiple-unit activity simultaneously with the EEG in chronic animals. Electroencephalography \& Clinical Neurophysiology, 1965, 19, 190-192.

\section{NOTE}

1. This research was supported by NSF Grant GB-7265 and by NIMH Grant MH-11150. 